undiagnosed. We identified factors associated with HIV test refusals among Black African sexually transmitted infection (STI) clinic attendees.

Methods Data on all STI clinic attendances in England in 2013 were obtained from the genitourinary medicine clinic activity dataset (GUMCADv2). Individuals diagnosed with HIV on or before $31^{\text {st }}$ December 2012 were excluded from analysis. Ethnic variations in HIV test refusal at an attendance were determined. Further analyses were restricted to Black Africans, and bivariate and multivariate associations between demographic and clinical characteristics and HIV test refusal were assessed. All associations were determined using generalised estimating equations regression and adjusted odds ratios (aORs) with 95\% confidence intervals (CIs) are reported.

Results Black Africans made 92,331 attendances at STI clinics in 2013 and refused an HIV test on 7,666 (8.3\%) occasions. After adjusting for gender/sexual orientation, Black Africans were least likely to have refused an HIV test [aOR (95\% CI): 0.503 (0.490-0.517)] (vs. White British persons). Among Black Africans, the odds of refusing an HIV test decreased with age [0.983 (0.980-0.986)] and were lower among those born outside the UK [0.676 (0.637-0.717)], those who were tested for HIV within the last year $[0.802(0.762-0.845)]$ and in men who have sex with men $[0.307(0.235-0.402)]$ (vs. heterosexual men and all women), while the odds were higher among those diagnosed with a new STI at the same attendance [1.272 (1.193-1.357)].

Conclusion Among Black Africans, targeted health promotion may be needed to improve HIV testing rates and decrease the proportion undiagnosed, especially for heterosexual men, women, younger, UK-born persons and those newly diagnosed with an STI.

Disclosure of interest statement There are no conflicts of interest.

\section{P17.06 HIV PREVALENCE AND FACTORS ASSOCIATED WITH HIV TESTING AMONG YOUNG PEOPLE (15-24 YEARS) IN TANZANIA: A SECONDARY ANALYSIS OF THMIS DATA}

1,2DJ Damian*, 1,2SE Msuya. 'Kilimanjaro Christian Medical University College (KCMU Co), Moshi, Tanzania; ${ }^{2}$ Kilimanjaro Christian Medical Centre (KCMC), Moshi, Tanzania

10.1136/sextrans-2015-052270.584

Background HIV remained to be a major public health problem affecting the lives and livelihood of many in Sub-Saharan Africa (SSA). Young people (15-24 years) accounted for $42 \%$ of new HIV infections in people aged 15 years and older; and about $80 \%$ of them live in SSA. Knowing HIV status among young people can be one of key strategies in reducing transmission. Knowing HIV status is associated with increased safe sex practices, decreased number of partners and early/timely access to ART in HIV-positive. In Tanzania, only 54\% of females and $34 \%$ of males aged $15-24$ years have ever been tested HIV with limited information regarding factors associated with testing among young people.

Methods This was a secondary analysis of Tanzania HIV and Malaria Indicator Surveys data (THMIS) conducted in 2011-12. Descriptive statistics, bivariate and regression modelling of survey data were used to determine predictors of HIV testing among young people in Tanzania.

Results Prevalence of HIV among young people is 2.0\% (young women $3 \%$ vs. young men $1 \%$ ). Nearly half of all young people have ever tested for HIV (44.9\%). HIV testing was associated with older age (20-24 years) $[\mathrm{AOR}=3.0,95 \% \mathrm{CI}: 2.4-3.6]$; female gender $[\mathrm{AOR}=2.9,95 \% \mathrm{CI}: 2.4-3.5]$; currently married $[\mathrm{AOR}=3.0,95 \% \mathrm{CI}: 2.3-3.8]$; formerly married $[\mathrm{AOR}=2.4$, 95\% CI: 1.6-3.6]; primary education $[\mathrm{AOR}=1.8,95 \% \mathrm{CI}$ : 1.3-2.5]; secondary education $[\mathrm{AOR}=3.5,95 \% \mathrm{CI}: 2.4-5.1]$; living in urban areas $[\mathrm{AOR}=1.4,95 \% \mathrm{CI}: 1.2-1.7]$ and having stigmatising attitudes towards HIV [AOR $=1.2$, 95\% CI: 1.0 1.4].

Conclusion HIV prevalence in young women is three times higher than in young men. In this high HI-prevalence setting almost half of young people have not tested for HIV. Provision of information and sensitisation among young people on HIV testing should focus on young people who are single, uneducated and living in rural areas.

\section{P17.07 SEXUAL RISK BEHAVIOUR PREDICTS MORE FREQUENT USE OF HIV SELF-TESTING: EARLY FINDINGS FROM THE FORTH TRIAL}

${ }^{1} \mathrm{R}$ Guy*, ${ }^{1} \mathrm{MS}$ Jamil, ${ }^{2} \mathrm{C}$ Fairley, ${ }^{1} \mathrm{~K}$ Smith, ${ }^{1} \mathrm{~A}$ Grulich, IJ Bradley, ${ }^{1} \mathrm{~J}$ Kaldor, ${ }^{2} \mathrm{M}$ Chen, ${ }^{3} \mathrm{~A}$ McNulty, ${ }^{4} \mathrm{M}$ Holt, ${ }^{5} \mathrm{D}$ Ryan, ${ }^{6} \mathrm{C}$ Batrouney, ${ }^{7} \mathrm{D}$ Russell, ${ }^{1} \mathrm{~B}$ Bavinton, ${ }^{1} \mathrm{P}$ Keen, ${ }^{1} \mathrm{DP}$ Conway, ${ }^{1} \mathrm{H}$ Wand, ${ }^{1,8} \mathrm{G}$ Prestage, on behalf of FORTH Study Group. ${ }^{1}$ The Kirby Institute, UNSW Australia, Sydney, Australia; ${ }^{2}$ Melbourne Sexual Health Centre, Melbourne, Australia; ${ }^{3}$ Sydney Sexual Health Centre, Sydney Hospital Sydney, Australia; ${ }^{4}$ Centre for Social Research in Health, UNSW Australia, Sydney, Australia; ${ }^{5}$ ACON, Sydney, Australia; ${ }^{6}$ Victorian AIDS Council, Melbourne, Australia; ${ }^{7}$ Cairns Sexual Health Service, Cairns, Australia; ${ }^{8}$ Australian Research Centre in Sex Health and Society, La Trobe University, Melbourne, Australia

\subsection{6/sextrans-2015-052270.585}

Background Most HIV diagnoses in Australia are among gay and bisexual men (GBM), yet less than a quarter of higher-risk GBM are testing at the recommended frequency (3-6 monthly). In the context of a randomised trial of HIV self-testing (FORTH), we examined the predictors of more frequent use of HIV self-testing among higher-risk GBM.

Methods Participants in FORTH trial included higher-risk GBM ( $>5$ sexual partners or condomless anal intercourse in the past 3 months). The trial is being conducted over 12 months, and men in the intervention arm receive 4 self-tests (OraSure's OraQuick home test) at baseline and additional self-tests on request. Using data from the baseline and 6 month surveys, we used logistic regression to examine predictors of using $>2$ self-tests over 6 months among participants in the intervention arm.

Results Of the GBM ( $\mathrm{n}=154)$ in the intervention arm, 59\% reported in the baseline survey they had condomless anal intercourse with casual partners (CLAIC) in the past 6 months and $56 \%$ had a HIV test every six months. Men who reported CLAIC in the past 6 months were more likely to use $>2$ HIV self-tests in the first 6 months of the trial (odds ratio:2.8,95\% CI: 1.2-6.7). No other baseline survey factors were associated with $>2$ self-tests, including; demographics, testing frequency, likelihood to self-test in the future, and reported testing barriers (the process of getting tested is too much hassle, I don't like having to return for results, I don't want to go to a clinic/doctor to get tested).

Conclusion These findings indicate men who report sexual risk behaviour are more likely to increase their testing frequency through self-tests, which is a key HIV prevention goal. However there is also a need to ensure the longer window period of the OraQuick self-test is understood to avoid infections being missed. 
Disclosure of interest statement The research is funded through a NHMRC Program grant from the NHMRC and self-test kits were purchased from OraSure Technologies Inc. (Bethlehem, PA, USA). The Kirby Institute and the Centre for Social Research in Health receive funding from the Australian Government Department of Health.

\section{P17.08 USING GRINDRTM, A SMARTPHONE SOCIAL NETWORKING APPLICATION, TO INCREASE HIV SELF- TESTING AMONG MEN WHO HAVE SEX WITH MEN IN LOS ANGELES}

${ }^{1}$ E Huang*, ${ }^{1}$ RW Marlin, ${ }^{2} \mathrm{~A}$ Medline, 'SD Young, 'J Daniels, ${ }^{1}$ JD Klausner. 'David Geffen School of Medicine at UCLA; ${ }^{2}$ McGill University

\subsection{6/sextrans-2015-052270.586}

Introduction In Los Angeles County, about 25\% of men who have sex with men (MSM) are unaware of their HIV positive status. Using smartphone social networking applications (apps) to promote free HIV self-testing might help reduce common barriers for testing, including poor access, stigma, and fear of loss of confidentiality.

Methods We advertised free HIV self-tests on Grindr ${ }^{\mathrm{TM}}$, a smartphone geosocial networking app popular with MSM, from April 17 to May 29, 2014, and from October 13 to November 11, 2014. The advertisements linked users to http://freehivselftests. weebly.com/to choose a self-test delivery method: US mail, a drugstore voucher, or from a vending machine. African American or Latino MSM $\geq 18$ years old were invited to take a survey on testing experiences.

Results During the two waves of the campaign, the website received 16,328 unique visitors (average: 227 per day) and 667 self-test requests. Of those 667, 471 (71\%) were requests for mailed self-tests, 157 (23\%) were for vouchers, and 39 (6\%) were to use the vending machine. Among 112 (63\%) survey respondents of 178 invited, study-eligible participants, $64 \%$ were between 18-30 years old, 18\% were Black/African American, $77 \%$ reported at least one episode of condomless anal sex in the past three months, and 38\% last tested for HIV over a year ago or had never tested. One hundred six (95\%) reported using the self-test was easy; 4 persons reported testing HIV positive and all $4(100 \%)$ sought medical care.

Conclusion Free HIV self-testing promotion through Grindr ${ }^{\mathrm{TM}}$ resulted in a large number of HIV self-test requests. Users preferred US mail self-test delivery, found the self-tests easy to use, and sought medical care if testing positive. Future work should evaluate different smartphone apps and compare smartphone social networking app promotion of self-testing with other HIV testing services.

Disclosure of interest statement The authors have no conflicts of interest to disclose.

\section{P17.09 COST-EFFECTIVENESS OF HIV SELF-TESTING PROMOTION THROUGH GRINDRTM, A SMARTPHONE SOCIAL NETWORKING APPLICATION}

${ }^{1}$ E Huang*, 'RW Marlin, ${ }^{2} A$ Medline, 'SD Young, ${ }^{1} \mathrm{~J}$ Daniels, 'JD Klausner. 'David Geffen School of Medicine at UCLA; ${ }^{2}$ MCGill University

10.1136/sextrans-2015-052270.587
Introduction Currently, the cost per new HIV diagnosis in the United States is estimated at $\$ 17,700$. HIV self-testing promotion through smartphone social networking applications (apps) might present an affordable way to help improve case finding. We evaluated the cost-effectiveness of an HIV self-testing program that linked geo-targeted mobile advertisements to an online self-test request system.

Methods The HIV self-testing program was offered in Los Angeles from April 17 to May 29, 2014, and from October 13 to November 11, 2014. During those periods, we placed advertisements for free HIV self-tests on Grindr ${ }^{\mathrm{TM}}$, a smartphone geosocial networking app popular with men who have sex with men (MSM). Users were linked to http://freehivselftests.weebly. com/to submit self-test requests. African American and Latino MSM $\geq 18$ years old were asked if they used the self-test and what the result of the self-test was. Cost-effectiveness was measured by the cost per person tested and the cost per new case of HIV identified.

Results Through the two offerings of the program, an estimated 455 users received and used an HIV self-test. Among 112 (63\%) survey respondents of 178 invited, study-eligible participants who self-identified as not being previously diagnosed with HIV, 4 (4\%) reported testing HIV positive; all 4 (100\%) sought medical care. The total direct costs of the program incurred from two waves of advertising (US\$2,670), self-test purchases (US\$13,130 at US\$26 per test), and personnel time (US\$1,800) was US $\$ 17,600$. The cost per person tested was US\$39, and the cost per new case of HIV identified was US\$4,400.

Conclusion Free HIV self-testing promotion through $\operatorname{Grindr}^{\mathrm{TM}}$ is an effective and affordable means of identifying previously undiagnosed cases of HIV among African American and Latino MSM. Future work should compare advertising on different smartphone social networking apps and evaluate methods to confirm self-reported HIV test results and linkage-to-care activities.

Disclosure of interest statement The authors have no conflicts of interest to disclose.

\section{P17.10 HIV TESTING SELF-EFFICACY IS ASSOCIATED WITH HIGHER HIV TESTING FREQUENCY AND PERCEIVED LIKELIHOOD TO SELF-TEST AMONG GAY AND BISEXUAL MEN}

${ }^{1} \mathrm{MS}$ Jamil ${ }^{*},{ }^{1} \mathrm{~B}$ Bavinton, ${ }^{1} \mathrm{R}$ Guy, ${ }^{2,3} \mathrm{C}$ Fairley, ${ }^{1} \mathrm{~A}$ Grulich, ${ }^{4} \mathrm{M}$ Holt, ${ }^{1} \mathrm{~K}$ Smith, ${ }^{2,3} \mathrm{M}$ Chen, ${ }^{5} \mathrm{~A}$ McNulty, ${ }^{1} \mathrm{DP}$ Conway, ${ }^{1} \mathrm{P}$ Keen, ${ }^{1} \mathrm{~J}$ Bradley, ${ }^{6,7,8} \mathrm{D}$ Russell, ${ }^{1} \mathrm{~J}$ Kaldor, ${ }^{1,9} \mathrm{G}$ Prestage, on behalf of FORTH Study Group. 'The Kirby Institute, UNSW Australia, Sydney, Australia; ${ }^{2}$ Monash University, Melbourne, Australia; ${ }^{3}$ Melbourne Sexual Health Centre, Melbourne, Australia; ${ }^{4}$ Centre for Social Research in Health, UNSW Australia, Sydney, Australia; ${ }^{5}$ Sydney Sexual Health Centre, Sydney Hospital, Sydney, Australia; ${ }^{6} J a m e s$ Cook University, Townsville, Australia; ${ }^{7}$ University of Melbourne, Melbourne, Australia; ${ }^{8}$ Cairns Sexual Health Service, Cairns, Australia; ${ }^{9}$ Australian Research Centre in Sex Health and Society, La Trobe University, Melbourne, Australia

10.1136/sextrans-2015-052270.588

Introduction Regular testing of individuals at high risk of HIV is central to current prevention strategies, and crucial to decrease the time-to-diagnosis. Little research has been conducted on 'self-efficacy': the perceived ability to undertake HIV testing among gay and bisexual men (GBM). We examined self-efficacy in relation to HIV testing frequency and likelihood to self-test among GBM.

Methods Participants were HIV-negative GBM at increased risk of HIV ( $>5$ partners or any condomless anal intercourse in 